\title{
La microfinance et ses portraits : médiations paradoxales de la parole des « pauvres»
}

Microfinance and its Portraits: Mediating the Voices of the Poor

\section{Odile Vallée}

\section{(2) OpenEdition}

\section{Journals}

Édition électronique

URL : http://journals.openedition.org/edc/6781

DOI : $10.4000 /$ edc. 6781

ISSN : 2101-0366

Éditeur

Université Lille-3

Édition imprimée

Date de publication : 1 juin 2017

Pagination : 55-70

ISBN : 978-2-917562-17-8

ISSN : $1270-6841$

Référence électronique

Odile Vallée, «La microfinance et ses portraits : médiations paradoxales de la parole des "pauvres » », Études de communication [En ligne], 48 | 2017, mis en ligne le 01 juin 2017, consulté le 20 avril 2019. URL : http://journals.openedition.org/edc/6781 ; DOI : 10.4000/edc.6781 


\section{La microfinance et ses portraits : médiations paradoxales de la parole des " pauvres »}

Microfinance and its Portraits:

Mediating the Voices of the Poor

Odile Vallée

Audencia Business School - Département Communication \& Culture, Rn'B Lab, Chercheuse associée au GRIPIC (CELSA) - EA 1498

ovallee@audencia.com 
L'article analyse le cas de l'accès, multiplement médié, à l'espace public médiatique ouvert à la parole des " pauvres " par la Campagne du Sommet du microcrédit, réseau hétérogène d'acteurs qui engagent un plaidoyer actif en faveur de la reconnaissance du microcrédit comme outil efficace dans la lutte contre la pauvreté. La personne et la parole des "pauvres ", saisies dans la forme sémiotique du portrait, circulent médiatiquement. Les modes de représentation et leurs effets de sélection produisent des paradoxes de la visibilité et soulève la question d'une éthique de la représentation.

Mots-clés : Microcrédit/microfinance, pauvre, espace public, visibilité, représentation, portrait, paradoxe.
The Microcredit Summit Campaign is composed of a varied network of actors. Since 1997, it has been advocating the recognition of microcredit as a tool to fight poverty. Portraits of "poor" clients are widely used in Microcredit Summit Campaign documents to represent borrowers and to support the claim of the efficacy of microfinance. For those who are represented, these portraits, considered here as a semiotic form, provide mediated access to the public sphere. Yet, biased modes of representation, they produce paradoxes of visibility which raise the issue of an ethics of representation.

Keywords: Microcredit/microfinance, poor, public sphere, visibility, representation, portrait, paradox. 
Le microcrédit s'est développé, dans sa forme moderne, vers le milieu des années 1970. La pratique désigne une mécanique de prêts de petites sommes d'argent, contre intérêts, à des personnes démunies n'offrant aucune garantie, en vue d'établir une activité productive. Le microcrédit est devenu la microfinance au fil de la diversification des produits financiers proposés, de l'accroissement de ses acteurs et de son extension géographique. Le secteur compte aujourd'hui plus de 94 millions d'emprunteurs répartis dans plus de 80 pays et affiche un encours de prêts qui excède les 78 milliards de dollars ${ }^{1}$. Cette pratique économique et sociale, qui fut initiée à un niveau local, s'est progressivement inscrite dans le champ politique et a conquis la scène institutionnelle et médiatique internationale. L'année 2005 fut à cet égard déclarée "Année de la microfinance " par les Nations Unies. L'apothéose symbolique de cette trajectoire fut le prix Nobel de la Paix décerné en 2006 à Muhammad Yunus, son réputé fondateur bangladais, et aux emprunteuses de sa banque Grameen.

Autour des institutions de microfinance (IMF), qui sont au cœur du secteur et servent les clients, s'est formé un écosystème globalisé d'organisations supports. Parmi elles, la Campagne du sommet du microcrédit (que nous nommerons à présent " la Campagne »), consiste en un réseau international et hétérogène d'acteurs économiques, politiques et sociaux. Depuis une position énonciative complexe, ce réseau déploie un plaidoyer actif en faveur de la reconnaissance du microcrédit comme outil de lutte contre la pauvreté, et ce en mettant les femmes les plus pauvres au centre de son action et de sa rhétorique. Les femmes, cible privilégiée de la microfinance, représentent en effet $73 \%$ des emprunteurs. Des portraits photographiques d'emprunteuses originaires des "Pays du Sud ", souriantes depuis la devanture de leurs échoppes, ou les mini-récits en trois temps relatant le pouvoir transformateur d'un prêt reçu sur la vie de son récipiendaire sont des lieux communs des discours et des campagnes de communication des IMF. Parfois disqualifiés et taxés de "romantiques »(Karnani, 2009), ces lieux communs, en plus d'être durablement associés à la pratique du microcrédit, demeurent omniprésents dans les documents publiés par les acteurs du secteur.

Ainsi, depuis 1999, la Campagne publie annuellement des rapports d'activité nommés «État de la Campagne du sommet du microcrédit » (State of the Microcredit Summit Campaign Report), diffusés aux membres du réseau et à ses partenaires institutionnels et mis à disposition de tous les publics intéressés par la démarche. Ces rapports donnent à voir des portraits de femmes, à la fois visuels et narratifs, qui ont une structure quasi identique, et ce peu importe les personnes mises en scène et leur lieu d'énonciation. En étant produits et relayés par différentes instances sur une pluralité de supports, ils fournissent

1 Sources provenant de MixMarket.org (2011). Disponible sur : http://www. themix.org/mixmarket (page consultée le 19 novembre 2015). 
dès lors une représentation ${ }^{2}$ particulière de la pauvreté. La Campagne propose en effet une représentation, à double titre, des plus " pauvres ». Elle se veut, à travers la promotion de l'accès au crédit, la porte-parole et l'artisane de leur autonomisation économique et, en même temps, elle construit et met en circulation une image particulière de leur individualité et de leurs capacités.

L'analyse des productions discursives de la Campagne offre un angle intéressant pour problématiser la question de l'accès des groupes sociaux défavorisés à l'espace public, car cette organisation participe activement à la mise en visibilité internationale de figures de "pauvres ". Axel Honneth (2000) aborde l'espace public en termes de lutte pour la reconnaissance. II s'écarte de la perspective du consensus formulée par Habermas $(1978,1992)$ pour opérer le passage à une théorie du conflit qui tient compte de l'expérience sociale des individus, de leurs (absence de) ressources symboliques, des mécanismes et des formes variées de l'action collective. L'espace public n'est donc pas le lieu de la seule publicisation des énoncés langagiers. Pour entrer dans une dynamique publique d'argumentation, les individus doivent pouvoir se percevoir de manière positive (Voirol, 2003a). La question des luttes pour la reconnaissance permet donc de thématiser la variété symbolique des interactions entre groupes sociaux dominés - que sont les clientes cibles de la microfinance - et les institutions que ces groupes contribuent à construire et qui les façonnent en retour.

Le corpus d'analyse se compose de la version papier des quatorze rapports publiés par la Campagne de 1999 à 2014. Ce format permet de saisir, dans le temps long, les transformations des modes de représentation des femmes emprunteuses, très majoritairement mises en scène. L'ensemble de ces rapports cumule plus de quarante photographies (qui se trouvent en couverture) de femmes clientes du microcrédit et d'une vingtaine de mini-récits de vie d'emprunteuses qui circulent dans le corps des textes. Si le corpus ${ }^{3}$ ne permet pas d'analyser la mécanique et les parties prenantes engagées dans la formulation et le choix des visuels et des récits présentés, l'analyse sémio-discursive des rapports permet de décrire les caractéristiques des portraits mobilisés, de dégager leurs fonctions dans l'économie discursive de la Campagne et

2 La notion de "représentation " peut-être questionnée dans le sens politique d'un mandat délégué par les « pauvres » et/ou préempté par des organisations tierces. Selon la définition formulée par Marin (1994), elle peut être également mobilisée dans les sens transitif (re-présenter quelque chose, rendre un absent à la présence) et réflexif (se présenter représentant quelque chose). Car quand la Campagne, en tant que collectif d'organisations, parle au nom des « pauvres » et les rend visibles, elle parle aussi d'elle-même. Elle dévoile l'imaginaire (Charaudeau, 2007) et les « arrière-pensées » (Wrona, 2008) qui régissent sa stratégie sémio-discursive.

3 L'article exploite un corpus en langue anglaise constitué dans le cadre d'une thèse de doctorat en Sciences de l'information et de la communication qui interroge les processus de construction de la microfinance en tant que cause internationale. 
de pointer leurs limites. Ces dimensions sont essentielles au questionnement des normes qui régulent la mise en visibilité des emprunteuses pauvres. Elles dégagent les paradoxes soulevés par des formes sémiotiques qui influencent la formulation et le positionnement d'une revendication directe de visibilité par ces femmes. Les formes sémiotiques mobilisées régulent en effet des modes d'apparition, révèlent des imaginaires et des rapports de force symboliques entre les membres du réseau, les clients et les spectateurs de la pratique du microcrédit. L'analyse des portraits souligne dès lors les enjeux et les difficultés de l'accès multiplement médié à l'« espace public transnational » (Fraser, 2007) ouvert à ces « contre-publics subalternes » (Fraser, 2001) par des organisations «bien intentionnées ".

Ainsi, après avoir présenté la Campagne et l'enjeu que représentent les femmes pour le microcrédit, l'article s'intéressera aux caractéristiques et aux usages des portraits visuels et narratifs des clientes pauvres mobilisées dans les rapports d'activités produits par la Campagne. Cette analyse sémio-discursive mettra alors en évidence la mécanique qui permet à la Campagne de représenter un groupe dominé, pourtant hétérogène, sur le plan symbolique, et, par translation, sur le plan politique.

\section{1. \\ La Campagne : apparition d'une " voix » et d'une « voie"}

Dix ans avant l'obtention du Prix Nobel de la Paix, la Campagne fut créée à l'initiative de trois acteurs : Sam Daley-Harris, fondateur de l'organisation non gouvernementale (ONG) Results ${ }^{4}$ et directeur du réseau de 1997 à 2011, Muhammad Yunus et l'américain John Hatch, fondateur de l'IMF Finca au Pérou. Elle emprunte plusieurs aspects de sa mécanique de fonctionnement à Results et adopte, depuis sa création, le statut de projet de Results Educational Fund (REF). REF, entité sœur de Results, fut créée en 1983 et peut, à la différence de cette dernière, collecter des dons. Considérée comme une organisation de «citoyens ordinaires » (grassroots), Results mène des activités de lobbying auprès du Congrès américain pour lutter contre la faim dans le monde. L'acronyme, qui signifie "résultats », est à ce titre emblématique de sa méthode de travail. REF a, plus spécifiquement, une activité d'« identification et de promotion des

4 Results est une organisation non gouvernementale américaine fondée en 1980. Ses membres mènent des actions de lobbying auprès des membres du Congrès américain, forment les citoyens ordinaires à l'activisme et formulent un plaidoyer en faveur de politiques publiques et de législations contre la pauvreté. Results Educational Fund (REF) soutient et gère des projets de lutte contre la pauvreté. Les deux structures partagent les mêmes locaux et travaillent en synergie. 
solutions les plus efficaces de lutte contre la pauvreté $»^{5}$.

La Campagne est lancée officiellement sur un mode événementiel lors du sommet mondial tenu en 1997 à Washington D.C., sommet qui a rassemblé plus de 2900 participants issus de 137 pays. Ce réseau, à géométrie variable et fonctionnant sur un mode d'adhésion volontaire, comprend plus de 2000 acteurs originaires de tous les continents : IMF, représentants de gouvernement, banques commerciales, militants individuels, etc. À l'occasion de son premier sommet, les acteurs de la Campagne se sont assignés l'objectif d'atteindre 100 millions d'emprunteurs avec une offre de microcrédit à échéance de 2005. L'objectif a été ensuite renouvelé. Les instances de gouvernance de la Campagne, "entrepreneurs de cause » (Neveu, 2011), coordonnent de fait une entreprise de communication stratégique de grande ampleur. Elles travaillent à constituer l'enjeu de la dissémination du microcrédit en " problème public » (Gusfield, 1981). En effet, leurs actions transforment une série d'événements individuels disséminés - les initiatives de ses membres - en un phénomène global qui acquiert une existence objective.

La Campagne est composée de plusieurs " conseils " qui rassemblent chacun une catégorie de membres (praticiens, investisseurs, organisations supports, etc.). Le réseau affiche ainsi une volonté de gouvernance participative et revendique une mixité d'adhérents - chacun pouvant répondre d'une position particulière selon le type d'organisations, les ambitions et les contextes politiques et socio-économiques nationaux dans lesquels s'inscrivent leur activité - qu'il engage, moyennant des débats internes et des dissensions, dans la défense de la cause du microcrédit. Selon Voirol (2003b), une cause est toujours le résultat d'un processus discursif qui permet au « collectif » d'acteurs qui la formulent d'expliciter progressivement et de mettre en récit leur propre posture. Mis en circulation, ce récit, qui dessine l'ambition dudit collectif, identifie ses partenaires et qualifie ses opposants, permet aux revendications exprimées d'acquérir « un espace de visibilité » et de discussion (Voirol, 2003b). Le collectif est donc responsable des termes et des formes sémiotiques retenus pour engager la cause défendue dans le monde social, permettre l'avènement du débat et, en l'occurrence, du jugement. Les membres de la Campagne, engagés dans des dispositifs directs de discussion que sont les sommets, ou médiés par les rapports d'activité qu'ils éditent annuellement, négocient leurs divergences de pratiques et de points de vue sur les orientations du secteur d'activité. La charge de la preuve de la nécessité et de l'efficacité du microcrédit leur incombe. Ils doivent accompagner, documenter et médiatiser la croissance constante du nombre d'emprunteurs pauvres, la pertinence des actions entreprises et prendre position vis-à-vis des évolutions du secteur. Le discours du collectif en épouse donc les aléas. 
À l'euphorie des vingt premières années de développement ont succédé différentes crises, internationalement médiatisées. Les origines de ces crises sont variées et critiquent, par exemple, des pratiques de recouvrement nonéthiques et le surendettement d'emprunteurs qui a conduit à des suicides ou à des mouvements sociaux de protestation. Elles pointent également l'absence d'impact irréfutable de la microfinance sur l'amélioration des conditions de vie des emprunteurs (Banerjee et Duflo, 2012), dénoncent la surcommercialisation du secteur (Roodman, 2012) matérialisée par des entrées en bourses lucratives d'IMF (comme Compartamos au Mexique en 2007) ou questionnent l'intégrité de Muhammad Yunus ${ }^{6}$. L'institutionnalisation de la Campagne a reposé en partie sur la capacité de ses membres à absorber ces crises et à les recomposer pour en faire un terreau d'innovation qui renouvelle les propositions d'actions (Vallée, 2016). Le "Label d'excellence » (Seal of Excellency for Poverty Outreach and Transformation in Microfinance) lancé par la Campagne en 2011 pour protéger les clients, réguler les pratiques et qualifier l'orientation d'acteurs de la microfinance sur un continuum qui va de la lutte active contre la pauvreté à la « simple » inclusion financière, est un exemple de cette mécanique.

La position énonciative de la Campagne est donc complexe. Ce réseau élève une " voix » et propose une voie qui s'énoncent dans des documents édités et des événements commandités. La " voix » et la voie de la Campagne englobent une variété de territoires, de perspectives et de pratiques qui ne sont pas d'emblée conciliables. Le collectif s'exprime en faveur des pauvres et au nom d'un réseau d'organisations aux nationalités et aux échelles d'action hétérogènes. Déployées dans le monde, ces structures sont composées d'acteurs de terrain qui travaillent au contact direct des clients, mais également d'investisseurs institutionnels internationaux qui n'approchent ces derniers qu'à travers ce que l'on dit d'eux dans des résultats d'études ou des récits de vie et des portraits visuels. Ces représentations sont dès lors les médiations d'une mise en visibilité des clients de la microfinance pour des publics qui sont à la fois proches et distants. Elles orientent ainsi les grilles de lecture et de réception d'une réalité beaucoup plus complexe. Elles fabriquent et inscrivent les conditions et les normes de leur visibilité.

Olivier Voirol (2005) identifie quatre formes de visibilité à partir des usages faits de cette notion dans les sciences sociales : la visibilité formelle, la visibilité pratique, la visibilité médiatisée et la visibilité sociale. La première forme de visibilité souligne l'importance des procédures d'inscription qui matérialisent des pratiques qui, autrement, demeureraient fuyantes. La deuxième forme, qui n'intéresse pas directement notre propos, concerne les mécanismes d'ajustements mutuels nécessaires aux circulations et interactions dans les espaces publics urbains. La troisième forme intègre l'élargissement du domaine de visi-

6 Comme dans le documentaire de Heinemann Tom (2011). Caught in Microdebt, Danemark, Heineman Média. 
bilité que favorisent les médiations techniques (télécommunications, internet, etc.). Enfin, la visibilité sociale considère les différents modes de manifestation de l'attention publique, ses conditions de possibilités et ses relais. Ces catégories permettent de dégager quatre axes de recherche opératoires pour notre analyse. Le premier axe se penche sur les normes qui régulent le visible et ce qui mérite d'être vu. Le second se concentre sur les mécanismes de formulation et de positionnement d'une revendication de visibilité. Le troisième axe questionne les processus qui permettent de devenir visible et le dernier axe souligne les paradoxes qu'ils sont susceptibles d'engendrer. Voirol déplace ainsi la focale de la capacité de voir vers celle de l'attention. L'expérience sociale, dynamique et conflictuelle, reconfigure et hiérarchise les frontières du visible.

Nous pouvons remarquer que les manières de se rendre visible et de voir apparaissent mutuellement conditionnées. La problématique de la lisibilité s'ajoute à notre sens à celle de la visibilité analysée par Voirol. Cette seconde notion recouvre les conditions et les modes de compréhension et d'interprétation des termes et des formes soumises au débat. Si la notion de visibilité questionne les modes de représentation des acteurs, celle de lisibilité, dans une perspective herméneutique, interroge le caractère nécessairement matériel et déchiffrable de ces représentations. Processus paradoxaux de la visibilité et de la lisibilité, quoique omniprésents dans le discours de la Campagne, la « voix » et la « voie » des pauvres semblent rendues inaudibles par un dispositif qui capte et canalise leur parole. Celles-ci s'effacent au profit de la « voix » et de la « voie " qui leur sont offertes par le réseau. Dans quelle mesure la mise en portraits et en récit des micro-emprunteuses opérée par les acteurs de la Campagne ouvre-t-elle à leurs visages, mots et actes un accès paradoxal à I'« espace public transnational »?

\section{2. \\ Le microcrédit et la question de "l'empowerment " des femmes}

Par rapport aux hommes, les femmes se sont imposées comme étant la cible privilégiée de la microfinance. Les premiers projets de microcrédit, démarrés au Bangladesh, en Inde et en Amérique latine au cours des années 1970, orientent effectivement leurs efforts vers les femmes parce que, selon Yunus (1997), de tous les publics cibles possibles, cette catégorie de population apparait comme la plus irrémédiablement pauvre. Et, de plus, le manque de pouvoir économique des femmes se voit aggravé par les contraintes familiales, sociales et religieuses qui pèsent sur elles ainsi que par l'absence corolaire de pouvoir politique. Le public féminin, idéologiquement « évident » des projets de lutte contre la pauvreté, s'est progressivement transformé en une cible stratégique pour un nombre croissant d'IMF inscrites dans une logique « gagnant-gagnant » (Morduch, 2000). Cette logique combine, d'une part, la 
recherche de la viabilité et de la pérennité financières de l'institution garante et, d'autre part, la provision de services à une catégorie de clients nécessiteux qui ont empiriquement fait la preuve de leur engagement consciencieux dans les programmes de microfinance, de leur « docilité » et de leur aptitude à gérer et à rembourser les sommes prêtées (Bacqué et Biewener, 2015, 85-87). Ce ciblage est encouragé par les orientations des organisations internationales du système onusien, pourvoyeuses de fonds nécessaires au développement de la microfinance. Celles-ci ont effectivement à la fois intégré la question de l'empowerment ${ }^{7}$ (sous l'angle de l'autonomisation économique) et celle du genre dans leur rhétorique et programmes d'actions dans le tournant des années 2000 (Bacqué et Biewener, 2015). Le ralliement aux engagements de la $4^{\mathrm{e}}$ Conférence onusienne sur les femmes tenue à Pékin en 1995 constitue, d'ailleurs, une des lignes d'argumentation de la Campagne et sert son ambition de reconnaissance institutionnelle.

Linda Mayoux, consultante indépendante, identifie trois objectifs liés à la microfinance en direction des femmes : l'acquisition de l'autosuffisance financière ; la réduction de la pauvreté afin d'améliorer le bien-être individuel ; l'émancipation qui permet aux femmes, à travers des stratégies renforçant leur participation politique, "d'élaborer des stratégies de mobilisation sociale et de défendre l'équité entre les sexes au niveau macroéconomique » (Bacqué et Biewener, 2015, 86-87). La figure de l'emprunteuse qui apparaît dans les documents publiés par la Campagne, loin d'être anecdotique, hérite de ces enjeux.

Les emprunteuses sont représentées par la Campagne. Les formes sémiotiques qui les saisissent et les rendent visibles et lisibles ont un statut de médiation, notion que Jean Davallon, muséologue et chercheur en Sciences de l'information et de la communication (SIC), caractérise comme suit :

[...] Ce que fait apparaître le modèle de la médiation, c'est moins les éléments (l'information, les sujets sociaux, la relation, etc.) que l'articulation de ces éléments dans un dispositif singulier (le texte, le média, la culture). C'est au fond cette articulation qui apparaît comme le tiers (Davallon, 2004, 53).

Suivant cette perspective, les mises en portrait et en récit opérées par la Campagne sont des médiations qui matérialisent et font circuler la figure d'une pauvreté rendue surmontable par l'industrie de ses victimes. Cette figure se construit, devient opérante et circule à différentes échelles (locale, nationale et internationale). Le mode narratif s'avère un choix privilégié pour animer, organiser, rendre visible et lisible les clients (et les « effets » du microcrédit), et ce en mettant en scène la vie des emprunteurs/teuses pour un public com-

7 La notion d'empowerment, difficile à définir, articule, par sa racine anglophone, l'idée de pouvoir autant que celle des processus d'apprentissage pour y accéder (Bacqué et Biewener, 2010,6). 
posé d'acteurs du secteur de la microfinance et de tiers. Cette narration se double de portraits photographiques qui capturent visuellement, dans le flux de ces vies, des situations voulues représentatives. Les photos, en figeant ces situations, les valorisent. En ce sens, ces portraits sont une forme sémiotique relationnelle (Wrona, 2008). Le portrait croise, et donc problématise, dans sa matière les regards du portraitiste, du portraituré et du spectateur. Le portrait est, de manière privilégiée, utilisé pour représenter les puissants dont « il confirme ou réalise la domination » (Wrona, 2008, 6). A l'inverse, « [...] dans le cas du portrait [de l'individu] ordinaire, le tout l'emporte largement sur la partie; et la personne racontée voit ses caractéristiques individuelles évacuées par la représentation d'une catégorie qui le dépasse »(Wrona, 2008, 7). Nous constatons que la figure de l'emprunteuse émerge à l'intersection de quatre facteurs principaux articulés dans leurs portraits. Ces facteurs excèdent la seule personalité des individus représentés et entérinent une perspective normative et surplombante de leurs capacités et aspirations :

- I'appartenance à un genre,

- une situation de vie contrainte,

- la capacité d'action des individus et son actualisation dans l'esprit d'entreprise,

- des aspirations légitimes et socialement recevables à la « dignité ».

\section{3. \\ Modes de représentation, effets de sélection}

Dans l'économie du discours de la Campagne, les portraits peuvent adopter, simultanément ou successivement, le statut de preuve d'efficacité de la microfinance sur les conditions de vie matérielle des clients, de preuve de leur autonomisation économique - voire politique - par les effets dérivés d'un accès au crédit, et de source d'inspiration pour l'action. Les visuels retenus en couverture des rapports montrent à ce titre principalement des femmes, tournées vers leur ouvrage ou posant, souriantes, face à l'appareil. Elles ont les mains occupées à une tâche ou sont en train de présenter le fruit de leur activité. Les photos exposent différents types ethniques des "Pays du Sud ». Les femmes photographiées sont d'origine africaine, latino-américaine ou sont issues du sous-continent indien. Ce sont les zones du monde où les IMF capitalisent le nombre le plus important de clients. L'ordre d'apparition des types ethniques n'est pas figé. Ces photos, d'apparence anodine, soulèvent la question difficile de la représentation de l'autre et comporte dans leurs détails visuels une charge stéréotypique que Boëtsch et Villain Gandossi décrivent dans ces termes :

L'expérience donnée au corps ne se situe pas dans le seul registre de l'apparence physique mais en une série de poses intégrant à la fois morphologie, vêtements, langage et gestuelle. Un ensemble dans lequel chaque élément conforte les autres et qui finit - dans le cadre du 
stéréotype - par être le signe de la totalité (Boëtsch et Villain-Gandossi, 2001, 20).

Les vies représentées par la Campagne ${ }^{8}$ sont rendues exemplaires par le seul effet de leur mise en avant. Le montage des photos met en scène des réalités apparemment simultanées dans le temps et juxtaposées dans l'espace. II produit alors une métonymie du global guidée par une représentation ethnicisée et genrée, de fait restreinte, des emprunteurs. Les photos sont disposées selon deux procédés. Dans les premiers rapports, les images sont fusionnées. Les limites des photos sont indistinctes : celles-ci paraissent se fondre les unes dans les autres. Le procédé de mise en série apparaît à partir de 2009. Les photos sont juxtaposées et génèrent une galerie de portraits individués qui suggère l'auto-affirmation des sujets montrés. Si le principe de la série indique un réservoir potentiellement inépuisable $d$ 'histoires positives équivalentes, l'absence de mention des sources des portraits d'une part, et la seule indication du prénom de ces femmes dans les récits d'autre part, engendrent un effet inverse d'anonymisation.

Les types ethniques des femmes photographiées en couverture sont similaires à ceux des vies racontées qui apparaissent alors comme leur prolongement. Les récits de vie circulent dans différents espaces au sein des rapports. Ils sont donnés à voir en épigraphe, en introduction, au sein d'une rubrique dédiée nommée "les visages derrière les statistiques " et en conclusion. De longueur variable, le récit de vie d'une emprunteuse, que son épilogue positif et la transformation émotionnelle du rapport de cette femme au monde qu'il affirme invitent à qualifier $d^{\prime}$ ' euphorique ", adopte une structure immuable et raconte une transformation. II décrit une situation de vie initiale, le moment fondateur du prêt et une situation de vie finale au contenu inversé. II met en scène des femmes originaires de tous les espaces non occidentaux qui racontent trois périodes structurantes de leur vie : un passé de souffrance, un présent de l'emprunt qui se renouvelle avec succès, et un futur enfin autorisé.

On repère deux régimes de narration qui s'organisent autour d'un point de vue externe à la vie racontée. La narration distanciée a l'apparence d'une étude de cas. Les marques d'énonciation du narrateur extérieur à l'histoire précisent des détails techniques et les propos de l'emprunteuse ne sont rapportés que de manière indirecte, comme le montre cet extrait d'un des rapports :

Mary Akoth vit à Ahero Town dans une des parties les plus pauvres de I'ouest du Kenya [...]. En 1992, Mary assista à une formation proposée par AFRICA NOW [...]. Aujourd'hui elle génère un profit de 8 dollars américains par jour (deux fois le montant de son premier prêt) [...]. Elle dit qu'elle

8 Les rapports de la Campagne sont archivés et consultables en ligne à l'adresse suivante : http// : http://www.microcreditsummit.org/resources.php (page consultée le 15 novembre 2015). À partir de 2013, les rapports font en effet l'objet d'une version multimédia en ligne. 
n'a plus honte [...] (Declaration \& Plan of Action - notre traduction, c'est nous qui soulignons).

La narration sous la forme de témoignage est également parfois utilisée. L'auteur de l'article cite, dans ce cas, directement l'emprunteuse mais peut également traduire et synthétiser certains de ses sentiments. Dans ces récits, l'énonciation est formulée, tout ou partie, à la première personne, à l'image de ces propos rapportés dans le State of the Microcredit Summit Campaign Report de 2002 : " 'Quand mes enfants pleuraient de faim la nuit, j'avais envie de me tuer', se rappelle Saraswathi Khrisnan, qui habite en Inde ».

Les vies racontées apparaissent en outre dépolitisées car les contextes sociaux, économiques et politiques dans lesquelles elles se déroulent ne sont jamais mentionnés. Dans ces récits, ces vies sont ramenées à une somme d'épreuves injustes et accablantes qui handicapent le quotidien. Le prêt d'argent connote alors la confiance, l'usage qui en est fait illustre le sens de l'initiative et l'épilogue positif forme l'espoir. La fin du récit de Saraswathi Khrisnan matérialise cela comme on peut le voir dans cet extrait du State of the Microcredit Summit Campaign Report de 2002 :

[...] Aujourd'hui la petite affaire de légumes de Saraswathi se porte bien grâce à son travail acharné et la formation dispensée par le programme. Elle est heureuse d'être capable d'offrir des opportunités à ses enfants. Avec les nouvelles sources de revenus de la famille, Saraswathi éprouve un sentiment de fierté et de sécurité qu'elle n'a jamais expérimenté avant. "Je ne mettrai plus mes enfants en gage ; ils seront éduqués. Maintenant, je veillerai à ce que mon mari soit bon et ne me frappe plus " (State of the Microcredit Summit Campaign Report, 2002 - notre traduction, c'est nous qui soulignons).

L'emphase sur les aspects " positifs » et l'aspiration à des valeurs normatives (la fierté, la sécurité ou encore la dignité, qui clôturent les narrations ainsi que la représentation des femmes au travail, construisent une figure d'emprunteuse méritante et entreprenante qui suggère une iconographie de la pauvreté industrieuse (Vallée, 2013). Cette fable morale rassemble les femmes pauvres des «Pays du Sud » sous les modalités d'un destin commun. Les spectateurs, qu'ils soient eux-mêmes issus de ces mêmes pays ou Occidentaux, se voient proposer un engagement de pair à pair nourri par la perception d'une égalité universelle d'aspirations - sinon de positions - entre eux et les plus démunis, et celle de l'utilité de la pratique du microcrédit directement mesurable à l'aune indicielle de la matière photographique (Barthes, 1980).

Cette galerie positive de portraits féminins produit cependant des effets de sélection qui stigmatisent. Elle supprime les hommes de la représentation ou les disqualifie explicitement, à l'image de l'exemple énoncé plus haut qui dresse un profil négatif de l'époux de Saraswathi Khrisnan. La sélection assigne un type ethnique et un genre aux clients du microcrédit qui conforte sémiotiquement 
et justifie narrativement des orientations idéologiques et gestionnaires. Les représentations effacent les pauvres des "Pays du Nord » et occultent le type " caucasien ». Elles montrent des individualités sérialisées et non des collectifs organisés. De plus, l'argent est absent des photos. Son obtention n'est jamais une fin en soi. II n'est, dans les récits, qu'un moyen pour une fin considérée comme moralement désirable pour une population pauvre.

Suite aux différents scandales de la microfinance décrits plus haut, la Campagne fera un retour à une iconographie humanitaire plus classique, dans ses rapports de 2013 et 2014, respectivement intitulés "Vulnérabilité » et " Résilience ". Ils adoptent, en pleine page de couverture, le portrait d'une mère et de son enfant. Cette variante de la figure de madone, vierge à l'enfant que la tradition occidentale hérite de l'histoire de l'art, souligne une vulnérabilité occultée par le prisme de l'imaginaire entrepreneurial. L'arrivée de cette iconographie est précédée par l'introduction dans le rapport de 2012 d'un récit de type dysphorique. Sous cette forme, l'épilogue positif disparaît dans un premier temps et réapparaît moyennant un épisode de " prise de conscience " qui remet en avant la capacité d'apprentissage et le ressort des emprunteuses face à l'adversité. Ce récit, positionné dans la conclusion du rapport, se clôture par l'allégation suivante de la part d'une des emprunteuses : "J'ai appris une vraie leçon et ne prends plus de prêts d'un trop grand nombre d'IMF. Je prends moins, pas plus. Je m'en suis sortie et, vous savez, vous devez persévérer jusqu'à ce que vous y arriviez ". Ces variations formelles des portraits visuels et narratifs montrent la difficulté du travail de représentation. Elles matérialisent l'effort de reconnexion à une "réalité » de terrain qu'opèrent les acteurs de la Campagne et prétendent éviter l'écueil du figement des modes de représentation retenus.

\section{4. Conclusion : se constituer en public?}

La Campagne prend la parole au nom d'emprunteuses pour promouvoir le microcrédit en tant que cause. Le réseau donne ainsi la parole aux femmes bénéficiaires du microcrédit par l'entremise de formes sémiotiques mises en circulation au sein d'outils de communication stratégiques qui amplifient leur visibilité. Le plaidoyer visuel et narratif de la Campagne en faveur des " pauvres » aspire à témoigner et à rendre compte d'une "réalité » observée, d'attitudes, de comportements et d'aspirations auxquels le cadrage photographique et le récit de vie offrent des raccourcis commodes. Il cherche à mettre en valeur le caractère inédit de "ces réalités " pour des publics à la fois membres et tiers du secteur de la microfinance. Mais l'articulation entre l'ethnicité, le genre et l'entrepreneuriat informe les normes d'un visible stéréotypique, réducteur et ambivalent. Car, si la Campagne participe à la large circulation d'une figure alternative de la pauvreté, cette visibilité, voulue positive, a des effets paradoxaux qui handicapent sa lisibilité. La tension entre ce que l'on choisit 
de montrer et ce que, de fait, on repousse dans le hors champ, ramène les pauvres à la catégorie d'emprunteur et les emprunteurs à une figure féminine issue des "Pays du Sud ». Cette vignette annule la complexité des relations de pouvoir à l'intersection desquelles ces femmes, groupe dominé et hétérogène, se trouvent (Crenshaw, 2005). Elle occulte également l'historicité de ces relations, moteur de ce qu'Elsa Dorlin (2005) nomme une "épistémologie de la résistance ». Le stéréotype induit une dépossession de l'image, des maux et des mots des groupes ainsi qualifiés. Les formes sémiotiques mobilisées par la Campagne circulent et investissent l'« espace public transnational », se banalisent et produisent un substrat symbolique qui doit être mis en discussion. Il doit être réapproprié, dépassé ou effacé par des processus de subjectivation et de formation d'une identité politique des "pauvres ». Ces mécanismes identitaires ne peuvent exclure la négociation des termes d'une éthique de la représentation symbolique et politique avec les organisations « bien intentionnées » qui parlent en leur nom. 
Bibliographie

Arendt H. (1988 [1961]). Condition de l'homme moderne, Paris, Calmann-Lévy.

Bacqué M.-H. et Biewener C. (2015). L'empowerment, une pratique émancipatrice, Paris, La Découverte.

Banerjee A. et Duflo E. (2012). Poor economics : a radical rethinking of the way to fight global poverty, New York, PublicAffairs.

Barthes R. (1980). La Chambre claire: note sur la photographie, Paris, Éditions de l'Étoile Gallimard, Seuil.

Boëtsch G. et Villain-Gandossi C. (2001). « Les stéréotypes dans les relations Nord-Sud. Images du physique de l'autre et qualifications mentales ». In Hermès, n³0, p. 17-23.

Charaudeau P. (2007). « Les stéréotypes c'est bien, les imaginaires c'est mieux ». In Boyer H. (dir.), Stéréotypage, stéréotypes : fonctionnements ordinaires et mises en scène, Paris, L'Harmattan.

Crenshaw K. (2005). " Cartographie des marges : intersectionnalité politique de l'identité et violence contre les femmes de couleur ", trad. C. Bonis. In Cahiers du genre, $n^{\circ} 39$, p. 51-82.

Davallon J. (2004). « La médiation : la communication en procès ? ». In MEl, n 19, p. 37-59.
Dorlin E. (2005). « De l'usage épistémologique et politique des catégories de 'sexe' et de 'race' dans les études sur le genre ". In Cahiers du genre, $n^{\circ} 39$, p. 83-105.

Fraser N. (2001). « Repenser la sphère publique : une contribution à la critique de la démocratie telle qu'elle existe réellement ». In Hermès, $n^{\circ} 31$, p. 125-156.

Fraser N. (2007). "Transnationalizing the Public Sphere: On the legitimacy and Efficacy of the Public Opinion in the Post-Westphalian World". In Theory, Culture \& Society, $n^{\circ} 24(4)$, p. 7-30.

Gusfield J. (1981). The culture of public problems : drinking-driving and the symbolic order, Chicago, University of Chicago Press.

Habermas J. (1978). L'Espace public : archéologie de la publicité comme dimension constitutive de la société bourgeoise, Paris, Payot.

Habermas J. (1992). “ 'L'espace public', 30 ans après ». In Quaderni, n¹8, p. 161-191.

Honneth A. (2000). La Lutte pour la reconnaissance. $\mathrm{Pa}$ ris, Les éditions du Cerf.

Karnani A. (2009). « Romanticizing the Poor ", In Stanford Social Innovation Review. Disponible sur : http://www.ssireview.org/articles/ entry/romanticizing_the_poor (page consultée le 19 septembre 2016). 
Marin L. (1994). De la représen-

tation, Paris, Gallimard, Seuil.

Morduch J. (2000). "The Microfinance Schism ». In World Development, vol. 4, $n^{\circ} 28$, p. 617-629.

Neveu É. (2011). Sociologie des mouvements sociaux, Paris, La Découverte.

Quéré L. (1992). « L'espace public : de la théorie politique à la métathéorie sociologique $"$. In Quaderni, n¹8, p. 75-92.

Roodman D. (2012). Due diligence : an impertinent inquiry into microfinance. Washington D.C., Center For Global Development.

Vallée O. (2013). « La figure de l'emprunteuse ». In L'Expansion Management Review, vol. 4, n 151, p. 28-37.

Vallée O. (2016). « La Campagne du Sommet du Microcrédit : Construction d'une réputation entre députation et imputation ". In Bratosin S. et al. (coord.), Actualité scientifique en communication des organisations, Bucarest, Universitatii din Bucuresti, p. 295-311.

Voirol O. (2003a). « L’Espace public et les luttes pour la reconnaissance, de Habermas à Honneth ». In Barril C. et alii (dir.), Le public en action : Usages et limites de la notion d'espace public en sciences sociales, Paris, L'harmattan, p. 117-137.

Voirol O. (2003b). «Apparition d'une cause politique 'globale' et critique de la 'mondialisation'. L'Action Mon- diale des Peuples (AMP) ». Disponible sur : http://www.afsp.msh-paris.fr/ activite/groupe/germm/collgermm03txt/germm03voirol.pdf (page consultée le 19 septembre 2016).

Voirol O. (2005). " Les luttes pour la visibilité. Esquisse d'une problématique ». In Réseaux, $n^{\circ} 129-130$, p. 89-121.

Wrona A. (2008). « L'ordinaire en portraits : une forme impossible ? ». In Communication \& Langages, $n^{\circ} 158$, p. 3-12.

Yunus M. (1997). Vers un monde sans pauvreté. L'autobiographie du banquier des pauvres. Paris, J.C. Lattès.

Yunus M. (dir.). (1991 [1982]). Jorimon and the Others, Faces of Poverty. Dhaka, Moni Printers \& Packages Ltd. 